\title{
The Simulation of the Chirp-Z Transform Based on MATLAB GUI
}

\author{
Yongping Huang ${ }^{1}$ Caixia $\mathrm{Li}^{2}$ and Xiuli $\operatorname{Tian}^{3}$
}

Abstract The Chirp-Z transform (CZT) algorithm is a digital signal processing algorithm, it is applicable to the general case calculating limited width of the Ztransform along the spiral curve. The CZT is more flexible than the Fast Fourier transform (FFT) in calculating the frequency spectrum, so it is used in various fields such as radar ranging, power prediction and so on. But the implementation steps of the algorithm are difficulty to understand. This paper introduces the realizing process of CZT based on MATLAB GUI, Including the computational path of CZT, the main sequences and their simulation of CZT and FFT, it also presents the main program and simulation.

Keywords: Chirp-Z Transform · Fast Fourier Transform · MATLAB Graphical User Interface

\subsection{Introduction}

The discrete Fourier transform (DFT) is the only Fourier transform that is discrete in both the time and frequency domain, which is defined for finite-duration sequences. It is a basic means of signal and spectrum analysis frequently used by many researchers and manufacturers in the test and measurement fields. However it didn't vastly be used until the fast Fourier transform (FFT) [1] was created in engineering when the sequence length $N$ is large. The FFT is the most powerful algorithm for implementing DFT. The implementation of the FFT algorithm has several limitations [2].

\footnotetext{
${ }^{1}$ Yongping Huang $(\bowtie)$

College of Electronics and Information Engineering, Hebei University, Baoding, China e-mail: hyp2013@163.com

${ }^{2}$ Caixia Li

College of Electronics and Information Engineering, Hebei University, Baoding, China

${ }^{3}$ Xiuli Tian

College of Electronics and Information Engineering, Hebei University, Baoding, China
} 
1. The length $N$ of the FFT algorithm is a composite number. (The widelyused FFT size is an integer power of 2, for example 1024 or 2048 points [2].)

2. The input length $N$ and output length $M$ must be same.

3. The FFT evaluates the z-transform of a sequence of $N$ samples which is equal interval of the unit circle.

However the chirp z-transform (CZT) algorithm is more flexible in calculating the frequency spectrum. Using the CZT algorithm one can efficiently evaluate the $\mathrm{z}$-transform at $\mathrm{M}$ points in the z-plane which lie on circular or spiral contours beginning at any arbitrary point in the z-plane. The angular spacing of the points is an arbitrary constant, and $\mathrm{M}$ and $\mathrm{N}$ are arbitrary integers. But the implementation steps of the algorithm are difficulty to understand for students.

MATLAB is software with strong function, high efficiency and visualization; it quickly and easily provides the correct answer and graphics taking advantage of its digital processing toolbox.

This paper introduces the realizing process of CZT based on MATLAB, Including the calculation path of CZT, the main sequences and the comparison with the FFT; it also presents the main program and simulation.

\subsection{CZT Algorithm [3, 4]}

The z-transform of a finite-number $N$ of nonzero points $x(n)$ is defined as

$$
X(z)=\sum_{n=0}^{N-1} x(n) z^{-n}
$$

If we let

$$
z_{k}=A W^{-k} \quad k=0,1,2 \ldots . . M-1
$$

Where $M$ is an arbitrary integer and both $A$ and $W$ are arbitrary complex numbers of the form

$$
A=A_{0} \mathrm{e}^{\mathrm{j} \theta_{0}}
$$

and

$$
W=W_{0} \mathrm{e}^{-\mathrm{j} \phi_{0}}
$$

Then $\quad z_{k}=A_{0} \mathrm{e}^{\mathrm{j} \theta_{0}}\left(W_{0} \mathrm{e}^{-\mathrm{j} \phi_{0}}\right)^{-k}=A_{0} W_{0}^{-k} \mathrm{e}^{\mathrm{j}\left[\theta_{0}+k \phi_{0}\right]}$

Along the contour of (1.5), (1.1) becomes

$$
X\left(z_{k}\right)=\sum_{n=0}^{N-1} x(n) z_{k}^{-n}=\sum_{n=0}^{N-1} x(n) A^{-n} W^{n k} \quad k=0,1,2 \ldots \ldots . M-1
$$

Let us use the ingenious substitution, due to Bluestein [5]

$$
n k=\frac{1}{2}\left[k^{2}+n^{2}-(k-n)^{2}\right]
$$


for the exponent of $W$ in (1.6), this produces an expression

$$
X\left(z_{k}\right)=W^{\frac{k^{2}}{2}} \sum_{n=0}^{N-1}\left[x(n) A^{-n} W^{\frac{n^{2}}{2}}\right] W^{-\frac{(k-n)^{2}}{2}}
$$

If we form two new sequence $f(n)$ and $h(n)$ according to the equation

$$
\begin{gathered}
f(n)=x(n) A^{-n} W^{\frac{n^{2}}{2}} \quad n=0,1,2 \ldots . . N-1 \\
h(n)=W^{-\frac{n^{2}}{2}}
\end{gathered}
$$

Then $\quad X\left(z_{k}\right)=W^{\frac{k^{2}}{2}} \sum_{n=0}^{N-1} f(n) h(k-n) \quad k=0,1,2 \ldots . . M-1$

Bluestein employed the substitution of (1.7) to convert a DFT to a convolution as in Fig. 1.1

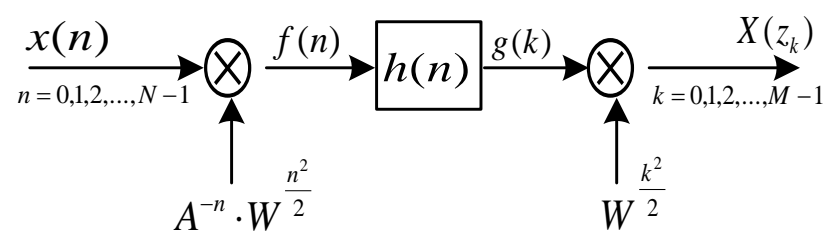

Fig. 1.1 The illustration of steps involved in computing values of the z-transform using the CZT algorithm

We call (1.5) is the computational path of the CZT.

According to Fig. 1.1, (1.11) can be thought of as a five-step process consisting of:

1. Choose $L, L=2^{m}$ and $L \geq N+M-1$

2. Form an $L$ point sequence $f(n)$ from $x(n)$, by

$$
f(n)=\left\{\begin{array}{lc}
x(n) A^{-n} W^{\frac{n^{2}}{2}} & 0 \leq n \leq N-1 \\
0 & N \leq n \leq L-1
\end{array}\right.
$$

Compute the L point DFT of $f(n)$ by FFT, call $F(k) \quad 0 \leq k \leq L-1$

3. Define an $L$ point sequence $h(n)$ by the relation

$$
h(n)= \begin{cases}W^{-\frac{n^{2}}{2}} & 0 \leq n \leq M-1 \\ \text { arbitrary } & M \leq n \leq L-N \\ W^{-\frac{(L-n)^{2}}{2}} & L-N+1 \leq n \leq L-1\end{cases}
$$

Or 


$$
h(n)= \begin{cases}W^{-\frac{n^{2}}{2}} & 0 \leq n \leq M-1 \\ W^{-\frac{(L-n)^{2}}{2}} & M \leq n \leq L-1\end{cases}
$$

Compute the $L$ point DFT of $f(n)$ by FFT, call $H(k) 0 \leq k \leq L-1$

4. $G(k)=F(k) H(k)$, compute the $L$ point IDFT of $G(k)$ by IFFT, call $g(n)$

5. Take first $M$ number of $g(n)$, call $g(k)$, multiply $g(k)$ by $W^{\frac{k^{2}}{2}}$ to give the desired $X\left(z_{k}\right)$

\subsection{Simulation of the CZT Based on MATALAB}

The simulation interface of the CZT based on MATALAB GUI is shown in Fig.1.2, it includes two parts: the Computational Path and the Simulation of CZT.

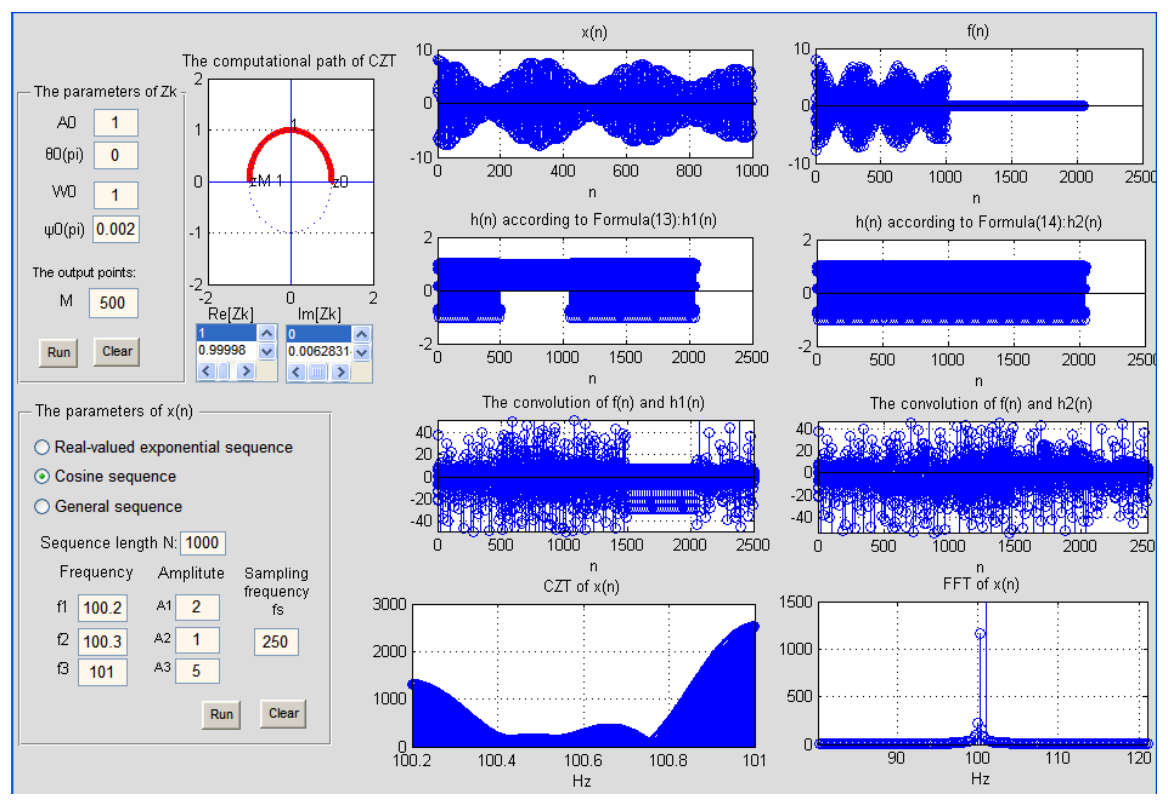

Fig. 1.2 The main simulation interface of CZT (cosine sequence) 


\subsubsection{The Computational Path of the CZT}

The simulation shows in the upper left corner of the Fig.1.2, if one inputs the parameters in (1.5), click the "Run" button, the path, the real and imaginary parts of $Z_{k}$ will generate in the interface. One will get different type path of the CZT by changing the parameters values. So we know that the $A_{0}$ and $\theta_{0}$ decide the starting point of $Z_{k}$ the $W_{0}$ decides the contour inward or outward, and the $\Phi_{0}$ decides the contour in a clockwise or counterclockwise rotation. There are some examples in Fig.1.3.

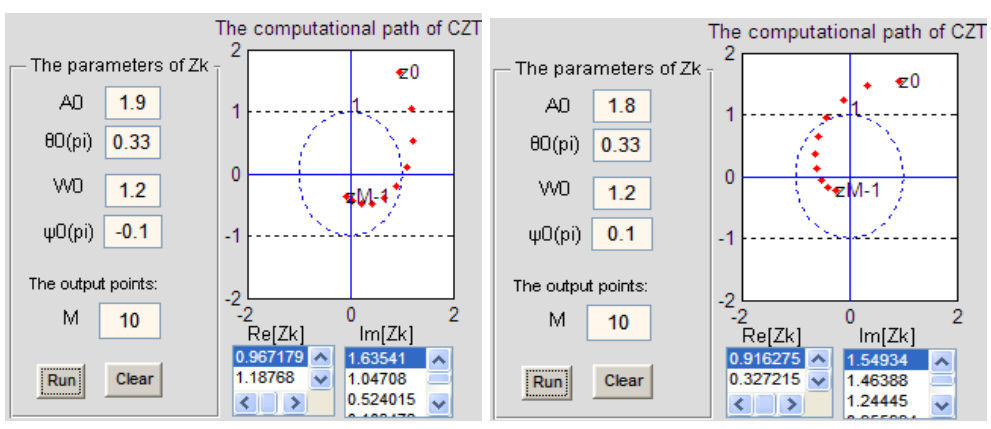

(a) $W_{0}>1 \quad \Phi_{0}<0$

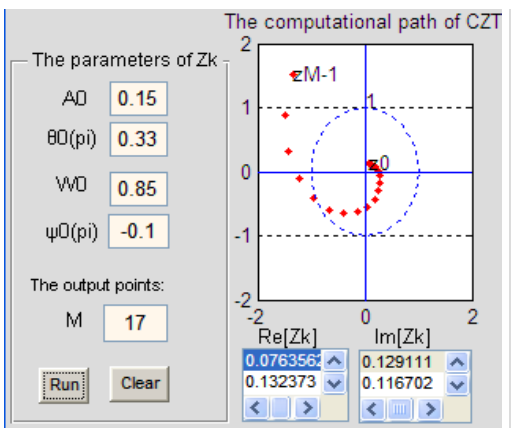

(c) $W_{0}<1 \quad \Phi_{0}<0$

(b) $W_{0}>1 \quad \Phi_{0}>0$

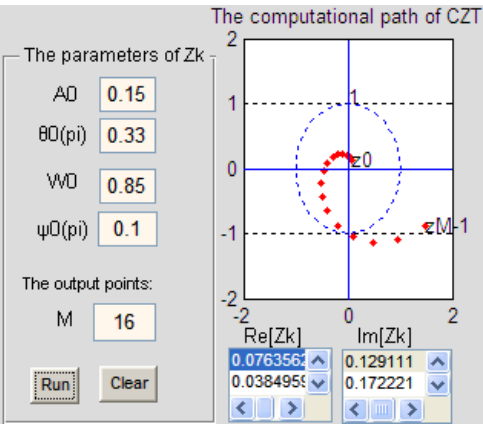

(d) $W_{0}<1 \quad \Phi_{0}>0$

Fig. 1.3 The computational path of CZT 


\subsubsection{The Simulation of CZT}

The simulation of CZT is shown in Fig.1.2 and Fig.1.4. We only provide three basic sequences which are real-sinusoidal sequence, cosine sequence and general sequence. One will get more simulation results easily by modifying the program. There are the sequences of $x(n), f(n), h(n)$ and the convolution of $f(n)$ and $h(n)$ in (1.12),(1.13)and(1.14) in the upper right corner of the Fig.1.2and Fig.1.4.The simulation of CZT and FFT are shown in the lower right corner. One can get the simulation results after select sequence and input its parameters. There are some examples in Fig.1.2 and Fig.1.4.

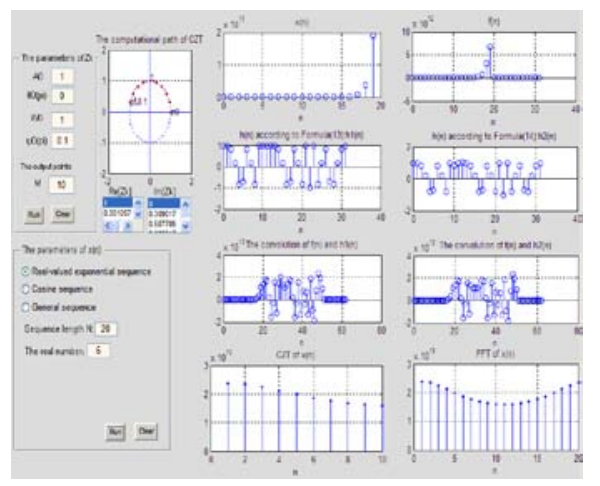

(a) real-sinusoidal sequence

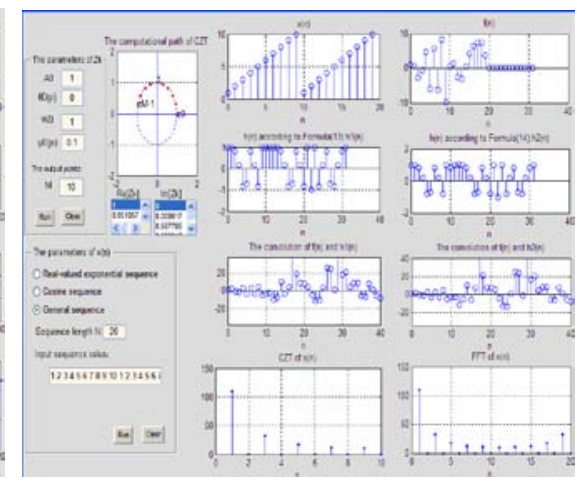

(b) general sequence

Fig. 1.4 Some simulation examples of CZT

One can understand that CZT is more flexible than FFT by comparing. The main program is shown as follows:

$\mathrm{L}=2^{\wedge}$ nextpow $2(\mathrm{M}+\mathrm{N}-1)$;

$\mathrm{A}=\mathrm{a} * \exp (\mathrm{j} * \operatorname{sita} 0) ; \mathrm{w}=\mathrm{b}^{*} \exp (-\mathrm{j} *$ fai0);

$\mathrm{n} 1=0.5 \cdot{ }^{*} \mathrm{n} . \wedge 2 ; \mathrm{w} 1=\mathrm{w} \cdot{ }^{\wedge} \mathrm{n} 1 ; \mathrm{A} 1=\mathrm{A} . \wedge(-\mathrm{n})$;

$\mathrm{fn}=[\mathrm{x} 1 . * \mathrm{~A} 1 . * \mathrm{w} 1, \mathrm{zeros}(1, \mathrm{~L}-\mathrm{N})]$;

$\mathrm{n} 2=-0.5 . * \mathrm{k} . \wedge 2 ; \mathrm{hn} 1=\mathrm{w} . \wedge \mathrm{n} 2 ; \mathrm{n} 3=-0.5 . *(\mathrm{~L}-\mathrm{k} 1) . \wedge 2 ; \mathrm{hn} 2=\mathrm{w} . \wedge \mathrm{n} 3$;

n31=-0.5.*(L-k11).^2; hn21=w.^n31;

h1=[hn1,ones(1,L-N-M+1),hn21]; h2=[hn1,hn2];

$\mathrm{y}=\mathrm{conv}(\mathrm{fn}, \mathrm{h} 1) ; \quad \%$ The convolution of $f(n)$ and $h(n)$ in (1.12) and (1.13)

$\mathrm{y}=\operatorname{conv}(\mathrm{fn}, \mathrm{h} 2) ; \quad \%$ The convolution of $f(n)$ and $h(n)$ in (1.12) and (1.14)

$\mathrm{y}=\mathrm{czt}(\mathrm{x} 1, \mathrm{M}, \mathrm{W}, \mathrm{A}) ; \quad \%$ compute CZT of $x(n)$

\subsection{Conclusions}

The advantages of simulation interface of CZT algorithm include the following: 
1. One can get the different simulation results by modifying the parameters in the interface which is designed.

2. Students can better understand the basic theory of CZT if it is used in class.

3. It can cultivate student's comprehensive ability and innovation ability if let them improve the interface and source program.

Acknowledgments This article is one of the initial results of the teaching reform project of College of Electronics and Information Engineering Hebei University "Digital Signal Processing quality course construction".

\subsection{References}

1. J. W. Cooley and J. W. Tukey (1965). An algorithm for the machine calculation of complex Fourier series. Math, Camp, 19, 297-301,

2. Tien T.Wang (1990). The Segmented Chirp Z-Transform and Its Application in Spectrum Analysis, IEEE Transactions on instrumentation and measurement, 39(2),318-323

3. Fenhua Li, Tieyuan Chang,etc (2007). Digital Signal Processing, Beijing, China Metrology Publishing House, 168-171.

4. L. R. Rabiner, R. w. Schafer and C. M. Rader(1969). The Chirp z-Transform Algorithm”, IEEE Transactions on audio and electroacoustics, AU-17(2), 86-92.

5. L. I. Bluestein(1968). A linear filtering approach to the computation of the discrete Fourier transform, NEREM Rec, 218-219 